Pacific Journal of Mathematics

RATIONAL HOMOLOGY AND WHITEHEAD PRODUCTS 


\title{
RATIONAL HOMOLOGY AND WHITEHEAD PRODUCTS
}

\author{
Michael Dyer
}

\begin{abstract}
D. W. Kahn defined a spectral sequence $\mathscr{C}(X ; R)$ for the Postnikov system $\mathscr{P}(X)$ of a 1-connected CW-complex which converges to $H_{*}(X ; R)$, the singular homology of $X$ with coefficients in $R$. We study $\mathscr{C}(X ; R)$ in two settings: (a) to give a generalization of the classical theorem of Eilenberg and MacLane concerning the dependence of $H_{i}(X ; Z)$ on the first nonzero homotopy group of $X(2.1)$ and (b) to give a complete computation of $H_{\imath}(X ; Q)(Q=$ rationals $)$ for $i \leqq 3 \cdot c(X)$ $(c(X)=$ connectivity of $X)$ in terms of the graded homotopy group $I \otimes Q=\left\{\pi_{\imath}(X) \otimes Q \mid 0<i \leqq 3 \cdot c(X)\right\}$ and the Whitehead product on this group $(0.1$ and 0.2$)$.
\end{abstract}

In $\S 1$ we give a quick description of $\mathscr{C}(X ; R)$ for later use and in $\S 2$ we generalize the Eilenberg-MacLane theorem by giving an exact sequence involving the first two nonzero homotopy groups. $\mathscr{C}(X, Q)$ is studied in $\S 3$, with the result that we are able to identify $E^{\prime}(X ; Q)$ somewhat above the diagonal (Kahn identified it below the diagonal in [7]) (3.3) and to show that the Whitehead product is the only non-zero differential operator, provided the total degree is less than $3 \cdot c(X)(3 \cdot 10)$. Section 4 gives the computations of $H_{i}(X ; Q)$ and various other applications.

1. Description of the Spectral Sequence of $\mathscr{P}(X)$. In this note $X$ is a $(n-1)$-connected space, $n>1$, having the homotopy type of a $C W$-complex. All maps and spaces are "pointed".

Let $\left\{X_{i}, r_{i}, \pi_{i}\right\}=\mathscr{S}(X)$ be a Postnikov system for $X$ (see [6] for definition). Choose $m>n$ and convert the map $r_{m}: X \rightarrow X_{m}$ into a fiber map. Use the same notation for the new map. In the tower of spaces

$$
X \stackrel{r_{m}}{\longrightarrow} X_{m} \stackrel{\pi_{m}}{\longrightarrow} X_{m-1} \stackrel{\pi_{m-1}}{\longrightarrow} \longrightarrow \cdots \stackrel{\pi_{n+1}}{\longrightarrow} X_{n}=K\left(\pi_{n}(X), n\right)
$$

$\pi_{\alpha} \circ \cdots \circ \pi_{m} \circ r_{m} \simeq r_{\alpha-1}(n+1 \leqq \alpha \leqq m)$. Let $r_{\alpha-1}$ denote this composition, $\alpha=n+1, \cdots, m$. Since all these maps are Hurewicz fibrations, $r_{\alpha-1}(\alpha-1<m)$ is a fiber map. Let $F_{i+1}=r_{i}^{-1}$ (base point) denote the fiber of $r_{i}: X \rightarrow X_{i}, i \leqq m$. The following is proved in [7].

Lemma 1.1. (a) $F_{i+1}$ is i-connected.

(b) $F_{i+1}$ is fibered over $K\left(\pi_{i+1}(X), i+1\right)$, with fiber $F_{i+2}$, via the map $r_{i+1} \mid F_{i+1}$.

(c) $X=F_{n} \supset F_{n+1} \supset \cdots \supset F_{m} \supset F_{m+1}$ is a finite de- 
creasing filtration of $X$.

For each $m$, the exact couple ([7]) $\mathscr{C}(\mathscr{P}(X), m ; G)$ is defined by

$$
\begin{aligned}
D_{r, s}^{1} & =\left\{\begin{array}{l}
H_{r+s}\left(F_{r} ; G\right), \text { if } r, s \geqq 0 . \\
0, \quad \text { otherwise, }
\end{array}\right. \\
E_{r, s}^{1} & = \begin{cases}H_{r+s}\left(F_{r}, F_{r+1} ; G\right), \text { if } r, s \geqq 0 . \\
0, & \text { otherwise, }\end{cases}
\end{aligned}
$$

where $G$ is any abelian group and $H_{*}$ is singular homology. If $D^{1}=$ $\sum_{\oplus} D_{r, s}^{1}, E^{1}=\sum_{\oplus} E_{r, s}^{1}$ then the couple maps $i: D^{1} \rightarrow D^{1}, j: D^{1} \rightarrow E^{1}$ and $k: E^{1} \rightarrow D^{1}$ are of bidegree (respectively) $(-1,1),(0,0),(1,-2)$. The bidegree of the differential operator $d_{i}: E^{i} \rightarrow E^{i}$ is $(i,-i-1)$.

In [7], Kahn shows that

$$
E_{j, s}^{1}=H_{j+s}\left(F_{j}, F_{j+1} ; G\right) \stackrel{q_{j *}}{\longrightarrow} \widetilde{H}_{j+s}\left(\pi_{j}(X), j ; G\right)
$$

is an isomorphism, provided $s \leqq j$, where

$$
q_{j}=r_{j} \mid F_{j}:\left(F_{j}, F_{j+1}\right) \rightarrow\left(K\left(\pi_{j}(X), j\right), *\right),
$$

thus indentifying the $E^{1}$ term below the diagonal.

2. Generalization of a theorem of Eilenberg-MacLane. In [4], Eilenberg and MacLane showed the dependence of the first few homology groups of a space $X$ upon the first nonzero homotopy group of $X$. We prove the following generalization.

THEOREM 2.1. Let $X$ be an $(n-1)$-connected space having the homotopy type of a $C W$-complex, $n \geqq 2$. Suppose $\pi_{i}(X)=0$ for $n<i<p$ and $p<i<q \leqq 2 n$. Then $H_{i}(X ; G) \approx H_{i}\left(\pi_{n}(X), n ; G\right)$ for $n \leqq i<p$ and any abelian group $G$. Furthermore, if we abbreviate $H_{\jmath}\left(\pi_{l}(X), l ; G\right)$ by $H_{j}(l ; G)$, we have the exact sequence

$$
\begin{gathered}
H_{q}(n ; G) \stackrel{\Phi_{q}}{\longrightarrow} H_{q-1}(p ; G) \stackrel{\psi_{q-1}}{\longrightarrow} H_{q-1}(X ; G) \stackrel{\chi_{q-1}}{\longrightarrow} H_{q-1}(n ; G) \stackrel{\Phi_{q-1}}{\longrightarrow} \cdots \\
\cdots \longrightarrow H_{i}(p ; G) \stackrel{\psi_{\imath}}{\longrightarrow} H_{i}(X ; G) \stackrel{\chi_{i}}{\longrightarrow} H_{i}(n ; G) \stackrel{\Phi_{i}}{\longrightarrow} H_{i-1}(p ; G) \longrightarrow \\
\cdots
\end{gathered}
$$

$\Phi_{i}=T_{i^{\circ}}(k)_{*}$, where $k: K\left(\pi_{n}(X), n\right) \rightarrow K\left(\pi_{p}(X), p+1\right)$ is the first $k$-invariant in a Postnikov decomposition of $X$ and $T_{j}: H_{j}\left(\pi_{p}(X), p+1 ; G\right) \rightarrow$ $H_{j-1}\left(\pi_{p}(X), p ; G\right)$ is the transgression, which is an isomorphism provided $0<j \leqq 2 p$. Further, $\psi_{p}$ is the Hurewicz homomorphism.

Proof. We consider $\mathscr{C}(X), m ; G)$ for $m>2 n . \pi_{i}(X)=0$ for $n<i<p, p<i<q$ implies by 1.1 (b) that 


$$
X=F_{n} \supset F_{n+1}=\cdots=F_{p} \supset F_{p-1}=\cdots=F_{q} \supset \cdots .
$$

Thus $E_{r, s}^{1}=0$ for $0 \leqq r<n, n<r<p, p<r<q$ and all $s$. This gives a two-term condition (see [5], chapter VIII) on the $E^{1}$-term of $\mathscr{C}(\mathscr{P}(X), m ; G)$. Using $(1.2)$ we have that $H_{i}(X ; G) \approx H_{i}\left(\pi_{n}(X), n ; G\right)$ for $n \leqq i<p$ (a 1-term condition here) and for $p \leqq i<q$ we have the exact sequence of the theorem. Note that we did not need $q \leqq 2 n$ in order to obtain the two-term condition, but only in order to use (1.2). It is clear from [7] that $\psi_{p}$ (the edge homonorphism) is the Hurewicz homomorphism.

We will now show that $\Phi_{i}=T_{i} \circ(k)_{*}$. Since $\Phi_{i}$ is essentially $d^{(p-n)}: E_{n, i-n}^{p-n} \rightarrow E_{p, i-1-p}^{p-n}([7])$, we will show that $d^{(p-n)}=T_{i}^{\circ}(k)_{*}$. As it has significance in its own right, we give it as a separate lemma.

Lemma 2.3 If $\pi_{i}(X)=0$ for $1 \leqq i<n, n<i<p, p<i<q$, then (a) $E_{r, s}^{!}=E_{r, s}^{p-n}$ for $r=n, p$ provided $s \leqq q-p$.

(b) The following triangle commutes for $s \leqq \min \{n, q-p\}$.

$$
E_{n, s}^{p-n}=\widetilde{H}_{n+s}(\pi_{n}(\underbrace{}_{\widetilde{H}_{n+s}}(X), n ; G) \stackrel{d_{p}^{p-n}}{\longrightarrow} \widetilde{H}_{n+s-1}(X), p+\overbrace{T}(X), p ; G)=E_{p,-(p-n)+s-1}^{p-n}
$$

where (i) $k: K\left(\pi_{n}(X), n\right) \rightarrow K\left(\pi_{p}(X), p+1\right)$ is the first k-invariant, (ii) $T$ is the composite $\partial \circ w_{*}^{-1}$

where $K\left(\pi_{p}, p\right) \subset P K\left(\pi_{p}, p+1\right) \stackrel{w}{\longrightarrow} K\left(\pi_{p}, p+1\right)\left(\pi_{p} \equiv \pi_{p}(X)\right)$ is the usual path space fibration. $T$ is an isomorphism provided $n+s \leqq 2 p$.

Proof. (a) follows because $\pi_{i}(X)=0$ for $1 \leqq i<n, n<i<p$

$$
\Rightarrow E_{n, s}^{1}=E_{n, s}^{p-n}
$$

for all $s$, since $d^{p-n}: E_{n, s}^{1} \rightarrow E_{p-(p-n)-1}$ is the first nonzero differential operator. $E_{p, s}^{1}=E_{p, s}^{p-n}$ provided $s \leqq q-p$ since $\pi_{i}(X)=0$ for $n<i<p$, $p<i<q$ implies that $d^{i}: E_{p-i s+i+1}^{i} \rightarrow E_{p s}^{i}$ is zero unless $i=p-n$ and $d^{i}: E_{p, s}^{i} \rightarrow E_{p+i, s-i-1}^{i}$ is zero provided $s \leqq q-p$.

(b) since $d^{p-n}$ is given by the composition (see 2.2)

$$
H_{n+s}\left(F_{n}, F_{p}\right) \stackrel{\partial}{\longrightarrow} \widetilde{H}_{n+s-1}\left(F_{p}\right) \stackrel{j_{*}}{\longrightarrow} H_{n+s-1}\left(F_{p}, F_{q}\right)
$$

we are asking that the following diagram commute:

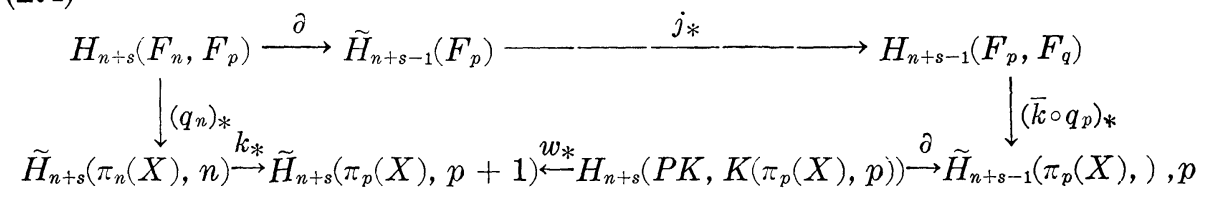


where $\bar{k}$ is defined by (2.6) below, and $q_{i}=\left.r_{i}\right|_{F_{i}}$ • (2.4) commutes if and only if

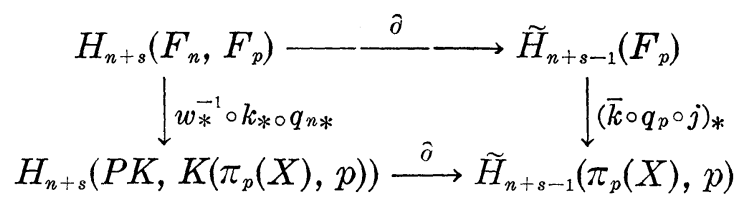

commutes. We have the following situation:

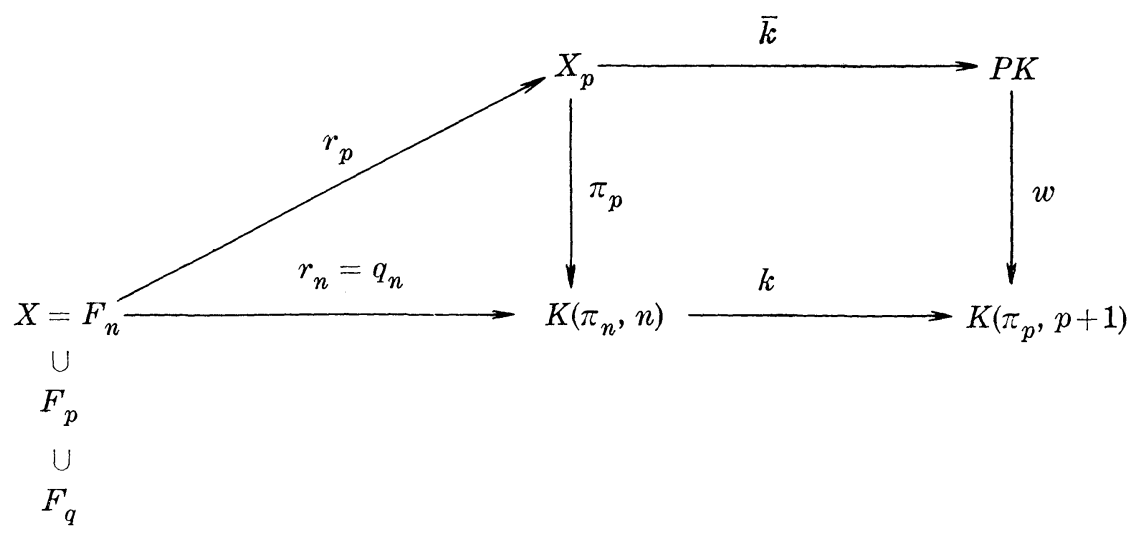

where $k \circ q_{n}=k \circ \pi_{p} \circ r_{p}=w \circ \bar{k}_{c} \circ r_{p} \Rightarrow w_{*}^{-1} \circ k_{*} \circ q_{n} *=\bar{k}_{*} \circ r_{p *} . \quad$ But $\left.\bar{k} \circ r_{p}\right|_{F_{p}}=$ $\bar{k} \circ q_{p}$ is clearly the same as $\vec{k} \circ q_{p} \circ j$ considered as maps of the pairs $\left(F_{p}, *\right) \rightarrow\left(F_{p}, F_{q}\right) \rightarrow(P K, *)$. This shows that (2.5) commutes.

By an argument similar to Lemma 2.3, we may identify the $d^{1}$ operator below the diagonal. This was claimed in [7], page 176.

Lemma 2.4. The following commutes for $s \leqq j$.

$$
\begin{gathered}
\widetilde{H}_{j+s}\left(\pi_{j}, j\right) \stackrel{d^{1}}{\longrightarrow} \widetilde{H}_{j+1}\left(\pi_{j+1}, j+1\right) \\
\left(k_{j} \circ i_{j}\right)_{*} \searrow \\
\widetilde{H}_{j+s}\left(\pi_{j+1}, j+2\right)
\end{gathered}
$$

where (a) $k_{j}: X_{j} \rightarrow K\left(\pi_{j+1}(X), j+2\right)$ is the $j$ th $k$-invariant,

(b) $i_{j}: K\left(\pi_{j}(Y), j\right) \subset X_{j}$ is the inclusion, and

(c) $T$ is the transgression (which is an isomorphism for $s \leqq$ $j+2$ ).

3. Rational homology and Whitehead products. In this section we consider Kahn's spectral sequence with coefficients in $Q$, the rationals. For this special case we are able to identify the $E^{1}$-term considerably above the diagonal. This occurs because for $Q$ coefficients, 
$H_{*}(\pi, n ; Q) \approx a$ Hopf algebra over $Q$ on $\operatorname{dim}_{Q}\left(\pi \otimes_{z} Q\right)$ generators of degree $n$.

In [8], J. P. Meyer demonstrated how to compute Whitehead products in $\pi_{*}(X)$ from a Postnikov system for $X$ and in [7], Theorem 9.1, D. W. Kahn used Meyer's results to show that a certain higher differential operator in $\mathscr{C}(X ; Q)$ is the Whitehead product. In the range of our identification, we show that this differential is the only nonzero differential operator. This allows a complete computation of $H_{i}(X ; Q), i \leqq 3 \cdot c(X)$, in terms of the homotopy groups of $X$ and the (rational) Whitehead products, where $c(X)$ is the connectivity of $X$.

DeFINITION 3.1. Let $G$ be an arbitrary $Q$-vector space and $p$ be a positive integer. The skew-symmetric tensor product $S_{p}(G)$ is defined as

$$
S_{p}(G)=\left(G \otimes_{Q} G\right) / R
$$

where $R$ is the subspace generated by $\left\{g_{i} \otimes g_{j}-(-1)^{p \cdot p} g_{j} \otimes g_{i} \mid g_{i}, g_{j} \in G\right\}$. Suppose $\nu=\operatorname{dim}_{Q} G$, and let $\Lambda(\nu, p)$ be the free commutative graded algebra over $Q$ on generators $\left(t_{1}, \cdots, t_{\nu}\right)$ where degree $t_{i}=p$ ( $\nu$ need not be finite).

$$
\Lambda(\nu, p) \approx\left\{\begin{array}{l}
Q\left[t_{1}, \cdots, t_{\nu}\right] \text { if } p \text { even } \\
E_{Q}\left(t_{1}, \cdots, t_{\nu}\right) \text { if } p \text { odd }
\end{array}\right.
$$

where $Q\left[t_{1}, \cdots\right]$ is the graded polynomial algebra over $Q, E_{Q}\left(t_{1}, \cdots\right)$ is the graded exterior algebra over $Q$, on generators $t_{1}, \cdots, t_{\nu}$ of degree $p$. Then it is easy to see that $S_{p}(G) \approx \Lambda(\nu, p)_{2 p}$, the $Q$-module of $\Lambda(\nu, p)$ in degree $2 p$.

Lemma 3.2. Let $G$ be an abelian group. Then $H_{2 p}(G, p ; Q) \approx$ $S_{p}(G \otimes Q)$.

Proof. This follows because $H_{*}(G, p ; Q)=\Lambda\left(\operatorname{dim}_{Q}(G \otimes Q), p\right)$.

THEOREM 3.3. Let $c(X)=n-1$, for $n \geqq 2 . \quad$ In $\mathscr{C}(\mathscr{P}(X), \infty ; Q)$, the $E^{1}$-term is given as follows $\left(\otimes\right.$ means $\left.\otimes_{Z}\right)$ : For all $p>0$,

$$
E_{p, q}^{1}(X ; Q) \approx\left\{\begin{array}{l}
\pi_{p} \otimes Q, \text { if } q=0 \\
0, \text { if } 0<q<p, \\
S_{p}\left(\pi_{p} \otimes Q\right), \text { if } q=p \\
\pi_{p} \otimes \pi_{q} \otimes Q, \text { if } p+1 \leqq q \leqq 2 p-2,
\end{array}\right.
$$

where $\pi_{i} \equiv \pi_{i}(X)$ (see Figure 3.1). 


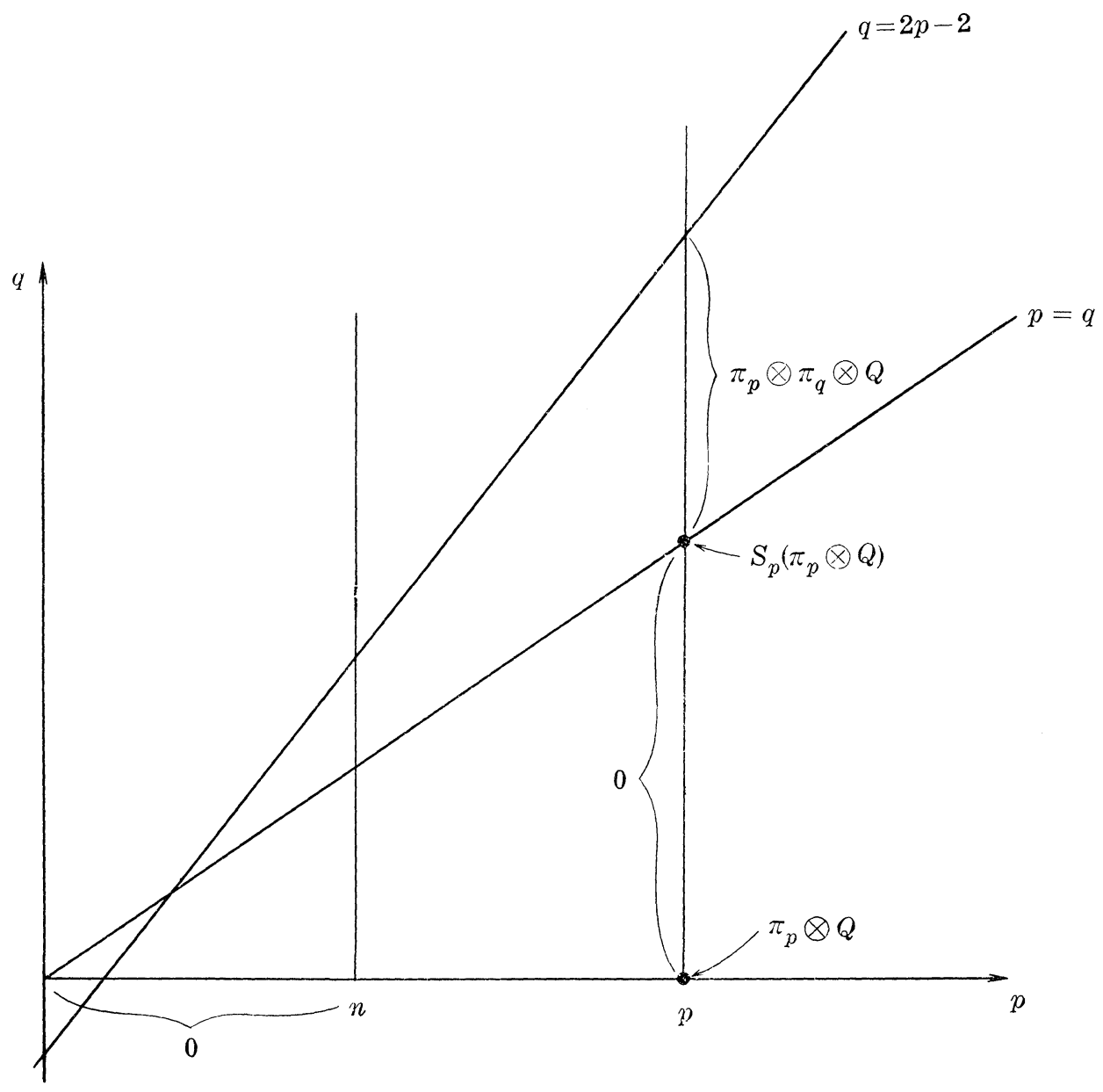

FIG. 3.1. $\quad E^{1}(X ; Q)$.

Proof. Let $p>1$ and consider the homology Serre spectral sequence [5] for the fibration $F_{p+1} \subset\left(F_{p}, F_{p+1}\right) \rightarrow\left(K\left(\pi_{p}, p\right)\right.$, *). The $E^{2}$-term, with coefficients in $Q$, is

$$
E_{r, s}^{2} \approx H_{r}\left(K\left(\pi_{p}, p\right),{ }^{*} ; H_{s}\left(F_{p+1} ; Q\right) \approx \tilde{H}_{r}\left(\pi_{p}, p ; Q\right) \otimes_{Q} H_{s}\left(F_{p+1} ; Q\right) .\right.
$$

Note that if $r<2 p$, then $E_{r, s}^{2}=0$ unless $r=p$ and

$$
E_{p, s}^{2} \approx \pi_{p} \otimes_{z} H_{s}\left(F_{p+1} ; Q\right) \text {. }
$$

It is easy to see from this, 1.1 (a), and the fact that

$$
H_{*}\left(\pi_{p}, p ; Q\right) \approx \wedge\left(\operatorname{dim}_{Q}\left(\pi_{p} \otimes Q\right), p\right)
$$

that 


$$
\begin{aligned}
& E_{p q}(X ; Q) \approx H_{p+q}\left(F_{p}, F_{p+1} ; Q\right) \\
& \quad \approx\left\{\begin{array}{l}
\pi_{p} \otimes{ }_{z} H_{q}\left(F_{p+1} ; Q\right), \text { if } 0 \leqq q \leqq 2 p-2, q \neq p . \\
H_{2 p}\left(\pi_{p}, p ; Q\right)=S_{p}\left(\pi_{p} \otimes Q\right), \text { if } q=p .
\end{array}\right.
\end{aligned}
$$

Now we show that if $p \leqq q \leqq 2 p-2$, then $H_{q}\left(F_{p} ; Q\right) \approx H_{q}\left(F_{q} ; Q\right) \approx$ $\pi_{q} \otimes_{Z} Q$. If $q=p$, then $H_{p}\left(F_{p} ; Q\right) \approx \pi_{p} \otimes Q$ by 1.1 (a) and the Hurewicz theorem. Consider the homology Serre spectral sequence with coefficients in $Q$ of the fibration $F_{p+1} \subset F_{p} \rightarrow K\left(\pi_{p}, p\right)$ given by 1.1 (b). If $p<q \leqq 2 p-2$, then the exact sequence of [5], page 284, implies that $i_{*}: H_{q}\left(F_{p+1}\right) \approx H_{q}\left(F_{p}\right)$. Similar arguments on the homology Serre spectral sequences for $F_{i+1} \smile F_{i} \rightarrow K\left(\pi_{i}, i\right), i=p+1, \cdots, q$ show that

$$
H_{q}\left(F_{p} ; Q\right) \approx H_{q}\left(F_{p+1} ; Q\right) \approx \cdots \approx H_{q}\left(F_{q-1} ; Q\right) \approx H_{q}\left(F_{q} ; Q\right) \approx \pi_{q} \otimes Q
$$

provided $p \leqq q \leqq 2 p-2$.

CoRollary 3.4. (Rational Hurewicz Theorem) If $i \leqq 2 c(X)$ then $h_{i} \otimes 1: \pi_{i}(X) \otimes Q \rightarrow H_{i}(X ; Q)$ is an isomorphism.

Proof. This is follows from 3.3 because the only non-zero term $E_{p, q}^{\prime}$ of total degree $i$ (for $i \leqq 2 c(X)$ ) is $E_{i, 0}^{1}=\pi_{i}(X) \otimes Q=E_{i, 0}^{\infty}$. Thus $\pi_{i}(X) \otimes Q \rightarrow H_{i}(X ; Q)$ is an isomorphism. Kahn's theorem 4.1 [7] identifies this map (the edge homomorphism) as $h_{i} \otimes 1$.

This result was known to Cartan and Serre in [2].

We will now study the differentials in $\mathscr{C}(X ; \infty ; Q)$. According to Theorem 2.2 of [3] (see also [9], Chapter 2), given $X, \exists$ a $C W$-complex $X \otimes Q$ and a map $f: X \rightarrow X \otimes Q$

(a) $\pi_{i}(X \otimes Q) \approx \pi_{i}(X) \otimes Q$

(b) $f$ is a homotopy equivalence modulo the class $\mathscr{S}^{-}$of torsion groups.

(c) $\exists$ an isomorphism $\nu$ such that the following commutes:

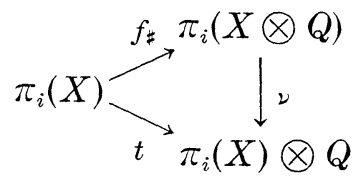

where $t(\alpha)=\alpha \otimes 1$, for $\alpha \in \pi_{i}(X)$.

Let $\widehat{X \otimes Q}$ be the space obtained from $X \otimes Q$ by killing off all the homotopy groups of $X \otimes Q$ in dimensions $\geqq 2 \cdot c(X)+1 ; i: X \otimes Q \rightarrow \widehat{X \otimes Q}$ the inclusion map. Consider the composite map $i \circ f: X \rightarrow \widehat{X \otimes Q}$. This induces an exact couple map from

$$
\mathscr{C}(\mathscr{P}(X) ; Q) \stackrel{\mathscr{C}(i \circ f)}{\longrightarrow} \mathscr{C}(\mathscr{P}(\widehat{X \otimes Q}) ; Q)
$$


which we shall see is an isomorphism in a certain range of dimensions on the $E^{1}$-term. Theorem 4.4 of [3] implies that all the $k$-invariants of $X \otimes Q$ are trivial, i.e.,

$$
\widehat{X \otimes Q} \cong \prod_{i=c(X)+1}^{2 \cdot c(Y)} K\left(\pi_{i}(X) \otimes Q, i\right) .
$$

This implies that the spectral sequence $\left\{E^{i}(\widehat{X \otimes Q} ; Q) ; \hat{d}^{i}\right\}$ collapses; i.e., all the $\hat{d}^{i}$ are zero. It follows from a theorem of Kahn [6], that $i \circ f$ induces maps $\mathscr{P}(i \circ f): \mathscr{P}(X) \rightarrow \mathscr{P}(\widehat{X \otimes Q})$ such that the following diagram commutes.

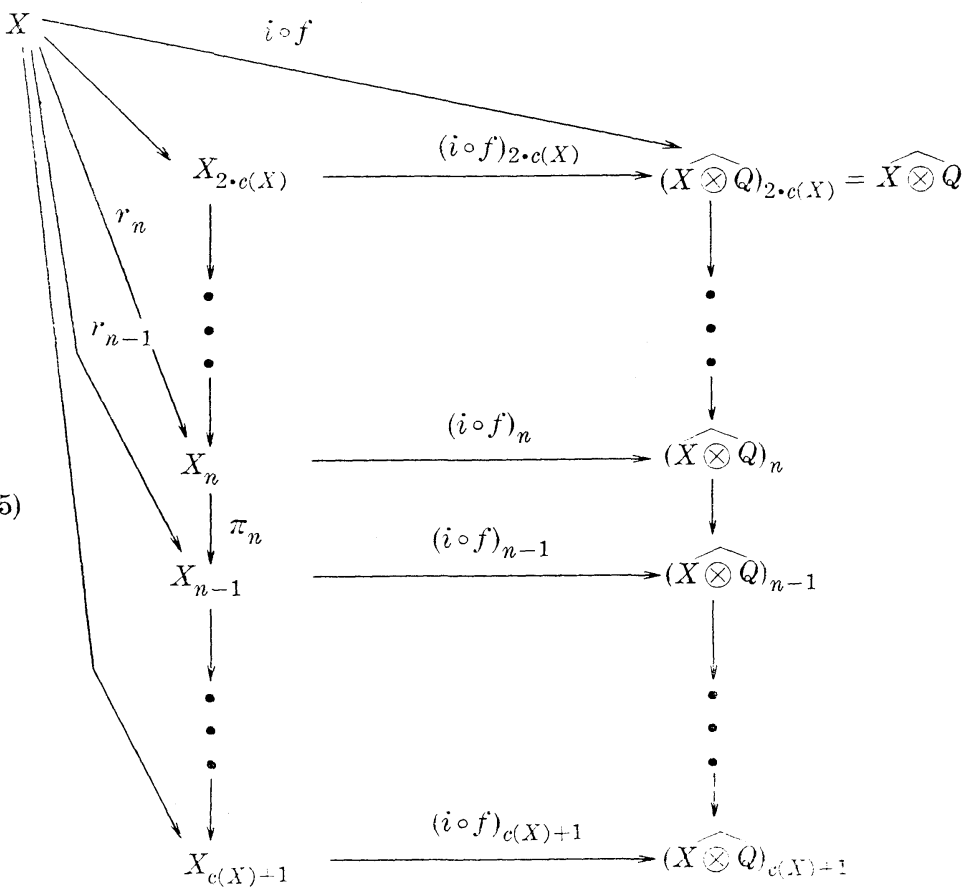

and $\pi_{i}\left(X_{n}\right) \stackrel{(i \circ f)_{\ddagger}}{\longrightarrow} \pi_{i}\left((\widehat{X \otimes Q})_{n}\right)(i>0)$ is an isomorphism mod $\mathscr{T}$. The commutativity of $(3.5) \Longrightarrow(i \circ f)\left(F_{n}(X)\right) \subset F_{n}(X \otimes Q)$ for $n \leqq 2 \cdot c(X)$. An easy induction using the mod $\mathscr{T}$ 5-Lemma [5], and the homotopy ladder induced by

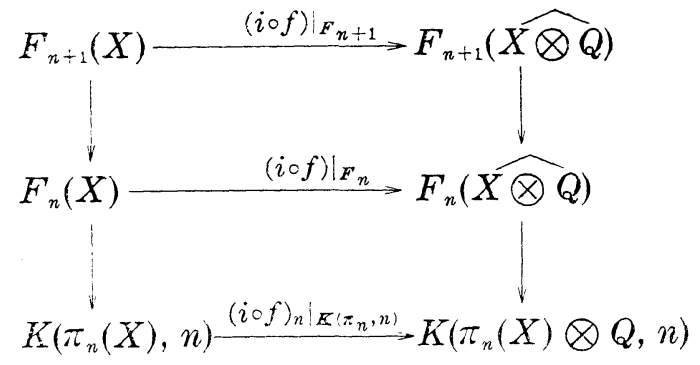


shows that $\left(\left.i \circ f\right|_{F_{n}(\mathrm{Y})}\right): H_{j}\left(F_{n}(X) ; Q\right) \rightarrow H_{j}\left(F_{n}(\widehat{X \otimes Q} Q) ; Q\right)$ is a $\mathscr{T}$-isomorphism for $j \leqq 2 \cdot c(X)$ (and an epimorphism for $j>2 \cdot c(X)$ ). By the Whitehead theorem $\bmod \mathscr{T}[5]$, page 512, we then have that

$$
\left(\left.i \circ f\right|_{F_{n}(X)}\right)_{*}: H_{j}\left(F_{n}(X) ; Q\right) \rightarrow H_{j}\left(F_{n}(\widehat{X \otimes Q}) ; Q\right)
$$

is an isomorphism for $j \leqq 2 \cdot c(X)$ and an epimorphism for $j=2 \cdot c(X)+$ 1 .

By the naturality of the universal coefficient theorem and the Serre spectral sequence, we have the following commutative diagram for $p \leqq 2 \cdot c(X)$ and $p<q \leqq 2 p-2$.

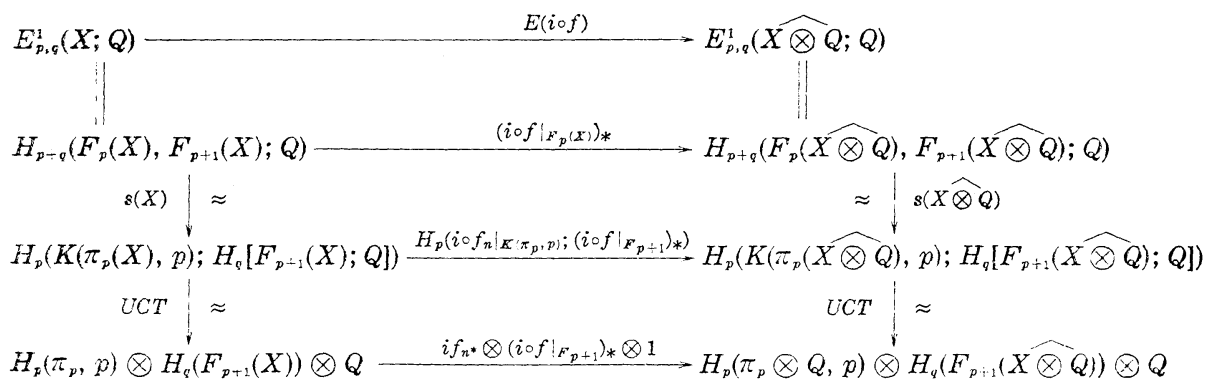

where $s(\cdot)$ in the above is the isomorphism defined from the Serre spectral sequence for $F_{p+1}(\cdot) \smile F_{p}(\cdot) \rightarrow K\left(\pi_{p}(\cdot), p\right)$. In this range of dimensions $(p \leqq 2 \cdot c(X), p<q \leqq 2 p-2)$ the vertical arrows are isomorphisms. 3.6 implies that the bottom row is an isomorphism, provided $q \leqq 2 \cdot c(X)$. A similar argument gives the case $q=p$.

From this we deduce that

$$
E^{1}(i \circ f): E_{p, q}^{\prime}(X ; Q) \rightarrow E_{p, q}^{\prime}(\widehat{X \otimes Q} ; Q)
$$

is an isomorphism provided $0 \leqq p \leqq 2 \cdot c(X), 0 \leqq q \leqq 2 \cdot c(X)$. See Figure 3.2. (3.8) implies

$$
E_{j, q}^{\prime}(X ; Q) \stackrel{E^{1}(i \circ f)}{\longrightarrow} E_{p, q}^{1}(X \widehat{\otimes Q} ; Q)
$$

is an isomorphism for $p+q \leqq 3 c(X)+1, p \leqq 2 c(X)$. (see Figure 3.2.) Assume now that $c(X) \geqq 2$. We will show that

$$
E_{p, q}^{i}=E_{p, q}^{1} \text { for } 2 \leqq i \leqq q-2
$$

whenever $c(X)+1 \leqq p \leqq 2 \cdot c(X), p \leqq q \leqq 3 c(X)-p$. (These are the only nonzero terms of total degree $\leqq 3 c(X)$ such that $q>0$. See shaded area in Figure 3.2.) Furthermore, all differential operators coming into $E_{p q}^{i}(i>0)$ are zero and all differential operators issuing 
forth from $E_{p, q}^{i}$ are zero except for $i=q-1$.

We show this by arguing on the total degree $j(2 c(X)+2 \leqq j \leqq$ $3 c X)$.

(a) $p+q=2 c(X)+2 \Rightarrow p=c(X)+1$. All differential operators with range $E_{c X+1 c X+1}^{i}$ are zero for $i>0$ 'since $E_{\mathrm{c} X+1-i c X+1+i+1}^{1}=0$ for all $i>0$. Similarly all $d^{i}: E_{c X+1, c_{X+1}}^{i} \rightarrow E_{c X+1+i, c X+1-i-1}^{i}$ are zero for $i \leqq$ $c(X)-1$ since the latter group is zero in that range.

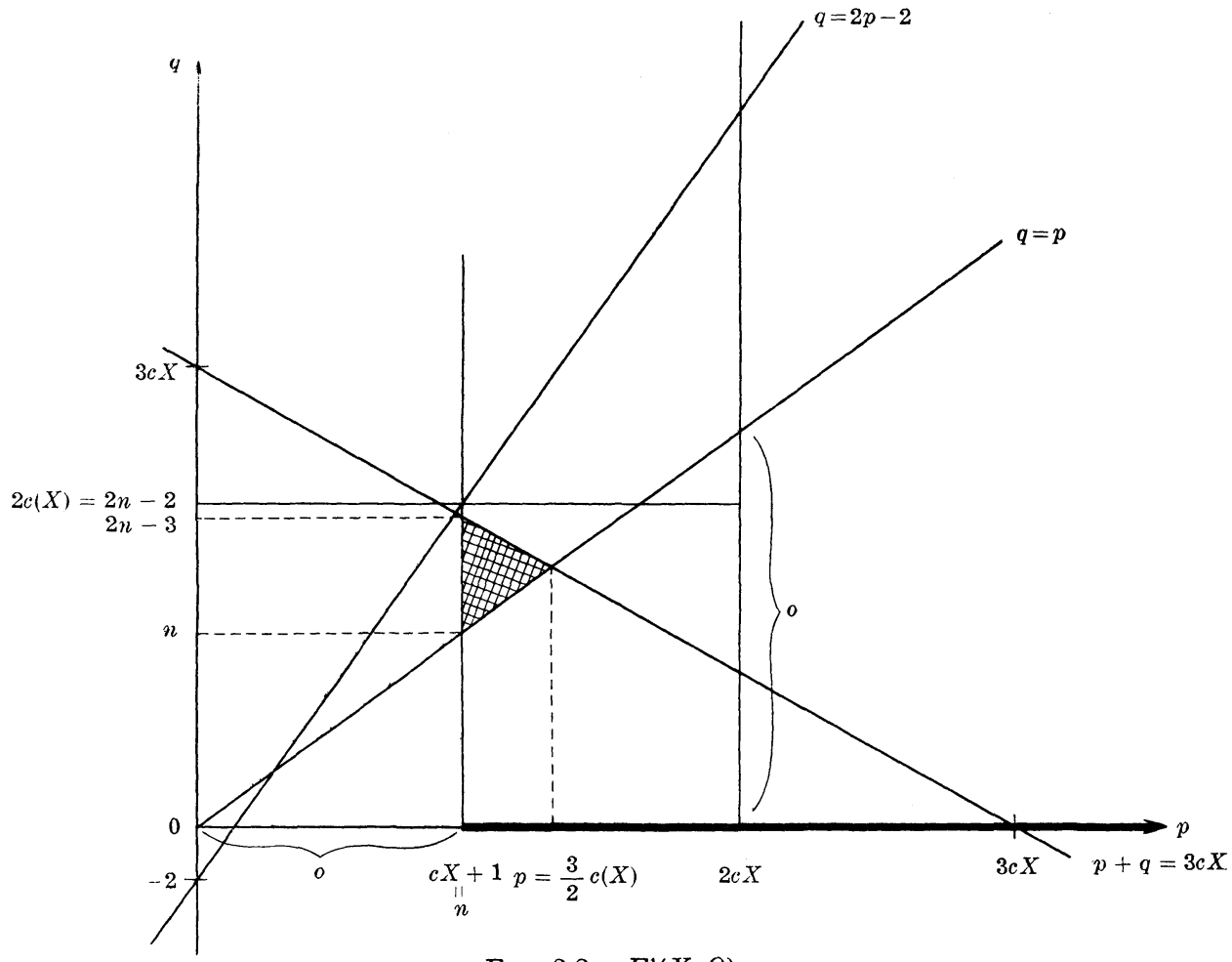

FIG. 3.2. $\quad E^{1}(X ; Q)$.

(b) Suppose $j>2 c(X)+2$. Consider $p+q=j \leqq 3 c(X)$, where $c(X)+1 \leqq p \leqq[j / 2]$, and the following commutative diagram

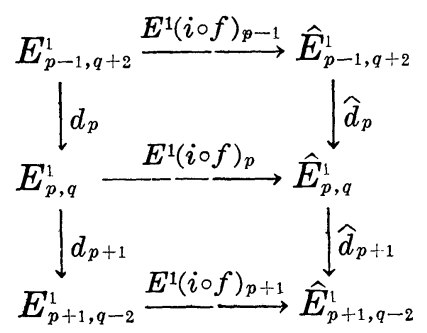

where $E^{1} \equiv E^{1}(X ; Q), \widehat{E}^{1} \equiv E^{1}(\widehat{X \otimes Q} ; Q) . \quad E^{1}(i \circ f)_{k}(k=p-1, p, p+1)$ is an isomorphism by 3.9 since the total degree in each case is $\leqq 3 c(X)+1$. 
Since $\hat{d}_{i}=0$, we have $d_{i}=0$ for $i=p, p+1$. Thus $E_{p, q}^{1}=E_{p, q}^{2}$ for $(p, q)$ satisfying the above. Similar arguments imply $E_{p, q}^{i}=E_{p, q}^{1}$ for $i=3,4, \cdots, q-2$.

(c) $d^{i}: E_{p, q}^{i} \rightarrow E_{p+1, q-i-1}^{i}$ is zero for $i>q-1$ since $q-i-1<0 \Rightarrow$ $E_{p+i, q-i-1}=0 . \quad d^{i}: E_{p-i, q+i-1}^{i} \rightarrow E_{p, q}^{i}$ is zero for $i \geqq q-1$ since $i \geqq q-1$, $q \geqq p \Rightarrow p-i \leqq p-q+1 \Rightarrow E_{p-i, q+i-1}^{1}=0$.

Thus the only (possibly) nonzero differential operator for each $(p, q)$ satisfying $c(X)+1 \leqq p \leqq 2 \cdot c(X), p \leqq q \leqq 3 c(X)-p$ is

$$
d^{q-1}: E_{p, q}^{q-1} \rightarrow E_{p+q-1,0}^{q-1}
$$

But this has been identified by Kahn in [7], Theorem 9.1, as the (rational) Whitehead product: If $q>p$

$$
\begin{gathered}
\pi_{p} \otimes \pi_{q} \otimes Q \stackrel{[,] \otimes i d}{\longrightarrow} \pi_{p+q-1} \otimes Q \\
\underset{\prod_{p, q}^{q-1}}{ } \approx \stackrel{d^{p-1}}{\longrightarrow} E_{p+q-1,0}^{q-1}
\end{gathered}
$$

or, if $q=p$

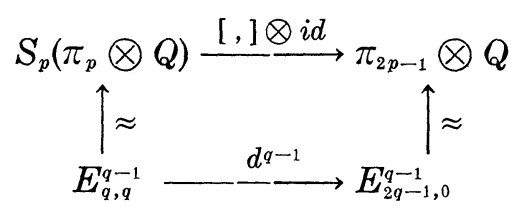

where $[$,$] is the Whitehead product.$

We have thus proved the following.

THEOREM 3.10. Let $c(X) \geqq 2$. If $p+q \leqq 3 \cdot(X)$ and $q \geqq p$, then (a) $d^{i}: E_{p-i, q+i+1}^{i} \rightarrow E_{p, q}^{i}$ is zero for all $i>0$.

(b) $d^{i}: E_{p, q}^{i} \rightarrow E_{p+i, q-i-1}^{i}$ is zero for $i=1,2, \cdots, q-2, q, q+1, \cdots$

(c) $d^{q-1}: E_{p, q}^{q-1} \rightarrow E_{p+q-1,0}^{q-1}$ is the rational Whitehead product.

4. Applications. We are now in a position to compute $H_{i}(X ; Q)$ $(i \leqq 3 \cdot c(X))$ completely in terms of the graded homotopy group $\Pi=$ $\left\{\pi_{i} \otimes Q \mid 1 \leqq i \leqq 3 \cdot c(X)\right\}$ and the rational Whitehead product on this group. For $i \leqq 2 \cdot c(X)$ this is given by the rational Hurewicz theorem (3.4). Let

$$
\operatorname{Ker}_{i j}= \begin{cases}\operatorname{Ker}\left\{\pi_{j} \otimes \pi_{i-j} \otimes Q \stackrel{[,] \otimes i d}{\longrightarrow} \pi_{i-1} \otimes Q\right\}, & c(X)<j \leqq\left[\frac{i-1}{2}\right] \\ \operatorname{Ker}\left\{S\left(\pi_{i / 2} \otimes Q\right) \stackrel{[,] \otimes i d}{\longrightarrow} \pi_{i-1} \otimes Q\right\}, & \text { if } i \text { even, } j=\left[\frac{i}{2}\right] \\ 0, & \text { if } i \text { odd, } j=\left[\frac{i}{2}\right] .\end{cases}
$$


and

$$
\operatorname{Ker}_{i}=\underset{c(X)<j \leq[i / 2]}{\bigoplus} \operatorname{Ker}_{i j} \quad(\oplus \text { denotes direct sum }),
$$

where [.] is the Whitehead product.

Furthermore, let

$$
\operatorname{Im}_{i j}= \begin{cases}\operatorname{im}\left\{\pi_{j} \otimes \pi_{i+1-j} \otimes Q \stackrel{[,] \otimes i d}{\longrightarrow} \pi_{i} \otimes Q\right\}, & \text { if } c(X)<j \leqq\left[\frac{i}{2}\right] \\ \operatorname{im}\left\{S\left(\pi_{(i+1) / 2} \otimes Q\right) \stackrel{[,] \otimes i d}{\longrightarrow} \pi_{i} \otimes Q\right\}, & \text { if } i+1 \text { even, } j=\left[\frac{i+1}{2}\right] \\ 0, & \text { if } i+1 \text { odd, } j=\left[\frac{i+1}{2}\right]\end{cases}
$$

and (since $\operatorname{Im}_{i j} \subset \pi_{i} \otimes Q$ for each $j$ )

$\operatorname{Im}_{i}=\sum_{c(x)<j \leq\lfloor(i+1) / 2]} \operatorname{Im}_{i j} \subset \pi_{i} \otimes Q .(+$ denotes sum, not necessarily direct)

Theorem 4.1. If $2 c(X)<i \leqq 3 \cdot c(X)$, then

$$
H_{i}(X ; Q) \approx \operatorname{Ker}_{i} \oplus\left(\pi_{i} \otimes Q / \operatorname{Im}_{i}\right)
$$

Proof. $3.4, \quad 3.10 \Rightarrow E_{i, 0}^{\infty} \approx\left(\pi_{i} \otimes Q / \operatorname{Im}_{i}\right)$ and $E_{p, q}^{\infty}(c(X)<p \leqq[i / 2]$, $p+q=i) \approx \operatorname{Ker}_{i p}$. These are the only nonzero terms of total degree $i$. Since all extensions split we have

$$
\begin{aligned}
H_{i}(X ; Q) & \approx E_{\imath, 0}^{\infty} \oplus \underset{c(X)<p \leq[i / 2]}{\bigoplus_{p, i-p}} E_{\mathrm{Ker}_{i} .}^{\infty} \\
& \approx\left(\pi_{\imath} \otimes Q / \mathrm{Im}_{i}\right) \oplus \mathrm{In} .
\end{aligned}
$$

Since Kahn [7] has identified the edge homomorphism with the Hurewicz homomorphism we see

THEOREM 4.2. If $i \leqq 3 \cdot c(X)$ and $h_{\imath} \otimes 1: \pi_{i}(X) \otimes Q \rightarrow H_{i}(X ; Q)$ is the Hurewicz homomorphism, then

(a) $\operatorname{Ker} h_{i} \otimes 1=\operatorname{Im}_{i}$

(b) $\operatorname{coker} h_{i} \otimes 1=$ Ker $_{i}$

Proof. This follows because $h_{i} \otimes 1$ is the natural map

$$
\pi_{\imath} \otimes Q \rightarrow \operatorname{Ker}_{i} \oplus\left(\pi_{\imath} \otimes Q / \operatorname{Im}_{i}\right) .
$$

COROLlaRY 4.3. If $i \leqq 3 \cdot c(X)$, then

(a) $h_{i} \otimes 1$ is a monomorphism $\Leftrightarrow \operatorname{Im}_{i}=0$

(b) $h_{i} \otimes 1$ is an epimorphism $\Leftrightarrow \mathrm{Ker}_{i}=0$. 
Note. By Proposition 2.1 (respectively, 4.1) of [1], $h_{i} \otimes 1$ is epic (respectively, monic) $\Leftrightarrow$ the $i^{\text {th }} k^{\prime}$-invariant ( $k$-invariant) of any homology (Postnikov) decomposition is of finite order. 4.3 gives another such characterization. This gives, for instance, the following theorem.

THEOREM 4.4 If $\pi_{i}(X ; Q)=0$ for $i>3 \cdot c(X)$, then all $k$-invariants are of finite order $\Leftrightarrow$ all rational Whitehead products vanish.

Finally, since it is usually easier to compute $H_{i}(X ; Q)$ than it is the Whitehead product, we will use these relations (4.1 and 4.2) to give information about the Whitehead products themselves.

THEOREM 4.5. Let $i \leqq 3 \cdot c(X)$ and consider the following statements:

(a) $\pi_{i} \otimes Q$ is generated by Whitehead products.

(b) For all $r$ such that $c(X)<r \leqq[(i-1) / 2], \pi_{r} \otimes \pi_{i-r} \otimes Q \rightarrow$ $\pi_{i-1} \otimes Q$ is injective.

(c) If $i$ even, $S\left(\pi_{i / 2} \otimes Q\right) \rightarrow \pi_{i-1} \otimes Q$ is injective. The following are true.

(d) $h_{i} \otimes 1=0 \Leftrightarrow(\mathrm{a})$

(e) $\operatorname{coker} h_{i} \otimes 1=0 \Leftrightarrow(\mathrm{b})$ and (c)

(f) $H_{i}(X ; Q)=0 \Leftrightarrow(\mathrm{a})$, (b) and $(c)$.

\section{REFERENCES}

1. M. Arkowitz, and C. R. Curjel, The Hurewicz homomorphism and finite homotopy invariants, Trans. Amer. Math. Soc., 110 (1964), 538-551.

2. H. Cartan, and J.-P. Serre, Espaces fibres et groupes d'homotopie, II, Applications,

C. R. Acad. Sci. Paris, 234 (1952), 393-395.

3. M. Dyer, Replacing Postnikov systems by simpler ones, (unpublished).

4. S. Eilenberg, and S. MacLane, Relations between homology and homotopy groups oJ spaces, Ann. of Math., (2) 46 (1945), 480-509.

5. S.-T. Hu, Homotopy Theory, Academic Press, New York, 1959.

6. D. W. Kahn, Induced maps for Postnikov systems, Trans. Amer. Math. Soc., 107 (1963), 432-450.

7. - The spectral sequence of a Postnikov system, Comm. Math. Helv., 40 (1966), 196-198.

8. J.-P. Meyer, Whitehead products and Postnikov systems, Amer. J. Math., 82 (1960).

9. D. Sullivan, Geometric Topology, Part I, Mass. Inst. of Tech. Notes, 1970.

Received October 5, 1970 and in revised form June 7, 1971.

UNIVERSITY OF OREGON 



\section{PACIFIC JOURNAL OF MATHEMATICS}

\section{EDITORS}

H. SAMELSON

Stanford University

Stanford, California 94305

C. R. HOBBY

University of Washington

Seattle, Washington 98105
J. DugundJI

Department of Mathematics

University of Southern California

Los Angeles, California 90007

RICHARD ARENS

University of California

Los Angeles, California 90024

\section{ASSOCIATE EDITORS}

E. F. BeCKENBACH

B. H. NeumanN

F. WOLF

K. YosHIDA

\section{SUPPORTING INSTITUTIONS}

UNIVERSITY OF BRITISH COLUMBIA

CALIFORNIA INSTITUTE OF TECHNOLOGY

UNIVERSITY OF CALIFORNIA

MONTANA STATE UNIVERSITY

UNIVERSITY OF NEVADA

NEW MEXICO STATE UNIVERSITY

OREGON STATE UNIVERSITY

UNIVERSITY OF OREGON

OSARA UNIVERSITY
UNIVERSITY OF SOUTHERN CALIFORNIA STANFORD UNIVERSITY

UNIVERSITY OF TOKYO

UNIVERSITY OF UTAH

WASHINGTON STATE UNIVERSITY

UNIVERSITY OF WASHINGTON

AMERICAN MATHEMATICAL SOCIETY

NAVAL WEAPONS CENTER

Printed in Japan by International Academic Printing Co., Ltd., Tokyo, Japan 


\section{Pacific Journal of Mathematics}

\section{Vol. 40, No. $1 \quad$ September, 1972}

Alex Bacopoulos and Athanassios G. Kartsatos, On polynomials

approximating the solutions of nonlinear differential equations........

Monte Boisen and Max Dean Larsen, Prüfer and valuation rings with zero

divisors ..........................................

James J. Bowe, Neat homomorphisms

David W. Boyd and Hershy Kisilevsky, The Diophantine equation

$$
u(u+1)(u+2)(u+3)=v(v+1)(v+2) \ldots \ldots \ldots \ldots \ldots \ldots \ldots
$$

George Ulrich Brauer, Summability and Fourier analysis ...............

Robin B. S. Brooks, On removing coincidences of two maps when only one,

rather than both, of them may be deformed by a homotopy ............

Frank Castagna and Geert Caleb Ernst Prins, Every generalized Petersen

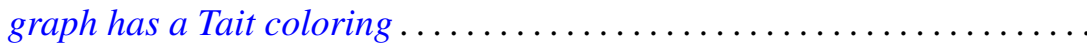

Micheal Neal Dyer, Rational homology and Whitehead products ..........

John Fuelberth and Mark Lawrence Teply, The singular submodule of a

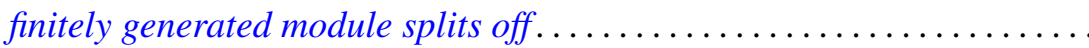

Robert Gold, $\Gamma$-extensions of imaginary quadratic fields ............ 83

Myron Goldberg and John W. Moon, Cycles in k-strong tournaments.......

Darald Joe Hartfiel and J. W. Spellmann, Diagonal similarity of irreducible

matrices to row stochastic matrices...............

Wayland M. Hubbart, Some results on blocks over local fields ..

Alan Loeb Kostinsky, Projective lattices and bounded homomorphisms....

Kenneth O. Leland, Maximum modulus theorems for algebras of operator

valued functions ...

Jerome Irving Malitz and William Nelson Reinhardt, Maximal models in the

language with quantifier "there exist uncountably many" ..

John Douglas Moore, Isometric immersions of space forms in space

forms.

Ronald C. Mullin and Ralph Gordon Stanton, A map-theoretic approach to

Davenport-Schinzel sequences ....................

Chull Park, On Fredholm transformations in Yeh-Wiener space. .

Stanley Poreda, Complex Chebyshev alterations ..............

Ray C. Shiflett, Extreme Markov operators and the orbits of Ryff. ...

Robert L. Snider, Lattices of radicals .....................

Ralph Richard Summerhill, Unknotting cones in the topological

category ................................

Charles Irvin Vinsonhaler, A note on two generalizations of $\mathrm{QF}-3 \ldots \ldots 229$

William Patterson Wardlaw, Defining relations for certain integrally

parameterized Chevalley groups...................

William Jennings Wickless, Abelian groups which admit only nilpotent

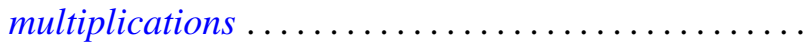

